\title{
fREDUCE: Detection of degenerate regulatory elements using correlation with expression Randy Z Wu${ }^{1}$, Christina Chaivorapol ${ }^{1}$, Jiashun Zheng ${ }^{1}, \mathrm{Hao} \mathrm{Li}^{* 1}$ and Shoudan Liang*2
}

Address: ${ }^{1}$ Department of Biochemistry and Biophysics, UCSF. 1700 4th Street, San Francisco, CA 94143-2542, USA and ${ }^{2}$ Department of Bioinformatics and Computational Biology, The University of Texas M.D. Anderson Cancer Center, 1515 Holcombe Blvd, Unit 237, Houston, TX 77030-4009, USA

Email: Randy Z Wu - rwu@genome.ucsf.edu; Christina Chaivorapol - cchaivor@genome.ucsf.edu; Jiashun Zheng - jiashun@genome.ucsf.edu; Hao Li* - haoli@genome.ucsf.edu; Shoudan Liang* - shoudan@mdacc.tmc.edu

* Corresponding authors

Published: 17 October 2007

BMC Bioinformatics 2007, 8:399 doi:10.1186/147|-2105-8-399
Received: 6 March 2007

Accepted: 17 October 2007

This article is available from: http://www.biomedcentral.com/I47I-2105/8/399

(C) 2007 Wu et al; licensee BioMed Central Ltd.

This is an Open Access article distributed under the terms of the Creative Commons Attribution License (http://creativecommons.org/licenses/by/2.0), which permits unrestricted use, distribution, and reproduction in any medium, provided the original work is properly cited.

\begin{abstract}
Background: The precision of transcriptional regulation is made possible by the specificity of physical interactions between transcription factors and their cognate binding sites on DNA. A major challenge is to decipher transcription factor binding sites from sequence and functional genomic data using computational means. While current methods can detect strong binding sites, they are less sensitive to degenerate motifs.

Results: We present fREDUCE, a computational method specialized for the detection of weak or degenerate binding motifs from gene expression or ChIP-chip data. fREDUCE is built upon the widely applied program REDUCE, which elicits motifs by global statistical correlation of motif counts with expression data. fREDUCE introduces several algorithmic refinements that allow efficient exhaustive searches of oligonucleotides with a specified number of degenerate IUPAC symbols. On yeast ChIP-chip benchmarks, fREDUCE correctly identified motifs and their degeneracies with accuracies greater than its predecessor REDUCE as well as other known motiffinding programs. We have also used fREDUCE to make novel motif predictions for transcription factors with poorly characterized binding sites.

Conclusion: We demonstrate that fREDUCE is a valuable tool for the prediction of degenerate transcription factor binding sites, especially from array datasets with weak signals that may elude other motif detection methods.
\end{abstract}

\section{Background}

Transcriptional regulation is modulated by a complex network of interactions between regulatory proteins and their binding targets on DNA. To comprehensively understand gene regulation at a systems level, a primary goal is to decipher the "regulatory code" that consists of knowledge of all transcriptional regulators, their DNA binding pro- files, and their regulatory targets [1]. Regulatory information can be inferred from the combined analysis of genomic sequence with an abundance of microarray based methods such as ChIP-chip (chromatin immunoprecipitation on microarray) [2,3] and transcription factor perturbation experiments $[4,5]$. However, highly reliable regulator specificies have been unattainable for many reg- 
ulators probed by such genomic-scale methods [1] since weak signals from regulators are often very difficult to isolate from experimental noise.

Thus, from a computational standpoint, a major challenge is to develop techniques that can extract maximal regulator specificity information from imperfect data. A common strategy among computational tools developed for this purpose is to first obtain a small group of genes in which a given motif may be statistically over-represented, from which the motif can then be elicited using methods such as position weight matrix updating and word enumeration [6-10]. While highly effective in some cases, a potential drawback of this approach is that the process of isolating a subgroup of sequences, typically done using clustering, cutoffs, or functional categorization, can be arbitrary. The delineation of signal from background may be poor for noisy experimental data, where cutoffs can lead to significant loss of information. Other algorithms, such as dictionary- [11] or steganalysis-based [12] methods, do not rely on clustering but can benefit from subgroup selection.

A technique used by many motif-finding algorithms is to integrate expression data into the search process [12-14]. For example, the algorithm REDUCE (Regulatory Element Detection Using Correlation with Expression) avoids subgroup selection in a natural way by genome-wide fitting of motif counts to expression data [15]. REDUCE is a deterministic method that first enumerates oligonucleotides and then identifies words whose occurrence in promoter sequences correlate most strongly with expression data. This procedure is applied iteratively to produce a set of oligonucleotides that produce the best simultaneous fit to the data. REDUCE requires only a single expression dataset and makes use of the entire genomic dataset (both signal and background) to assess the significance of individual motifs. This method, which has already been widely applied [16-21], allows greater sensitivity to weak transcriptional signals and facilitates the discovery of combinatorial effects between regulators.

One weakness of REDUCE is that it can miss weak but biologically significant variants of the regulator site. Highly degenerate motifs whose individual variants fall below the detection threshold will be missed altogether. This is particularly the case for regulators in higher mammalian genomes, which can exhibit strong site to site variation in specificity. Thus, we have generalized the REDUCE approach to examine words containing degenerate IUPAC symbols representing multiple bases (i.e. $\mathrm{S}=\mathrm{C}$ or G). However, a straightforward extension of REDUCE using exhaustive enumeration of degenerate motifs becomes impractical when the motif length or number of degenerate positions increase. Specifically, by including $m$ IUPAC symbols in a word of length $l$ the motif search space increases by a factor of $\frac{l !}{m !(l-m) !}\left(\frac{11}{4}\right)^{m}$ where 11 is the number of IUPAC symbols (excluding A,C,G,T). For example, the computational cost is increased by 340 -fold for $l=10$ and $m=2$, and by 3500-fold for $m=3$. Therefore, we have developed fast-REDUCE (fREDUCE), a significant re-implementation of the REDUCE algorithm that allows efficient searches of the extended space of degenerate motifs. We have applied fREDUCE to detect multiple motifs for transcription factor binding sites in yeast as well as human.

\section{Results Algorithm}

The original version of REDUCE identifies motifs by exhaustively correlating all oligonucleotides up to length $l$ in promoter sequences with expression data. However, the direct computation of the Pearson correlation coefficient is computationally laborious and is not well suited for analyzing large spaces of degenerate oligonucleotides. fREDUCE uses the following strategy to efficiently compute the Pearson coefficients of the most significant degenerate motifs (Figure 1): 1) A list of degenerate motifs that can be derived from the sequence data is generated. 2) For each degenerate motif, we can quickly compute a "pseudo-Pearson" coefficient, an estimate of the actual Pearson coefficient. The pseudo-Pearson coefficient is guaranteed to be an upper-bound on the actual Pearson coefficient and is used as a filter to eliminate most (typically $>99.9 \%$ ) of the motif list. 3) Actual Pearson coefficients are computed and the top motif is found and 4) The contribution from the top motif is subtracted from the expression data to form a residual, which is used for subsequent rounds of motif searching.

\section{Performance Assessment with Yeast ChIP-chip}

To assess the performance of fREDUCE, we applied the algorithm to 352 ChIP-chip experiments from Harbison et. al. [1] involving 203 known and putative transcription factors in the budding yeast $S$. cerevisiae. For each ChIPchip experiment, we correlated the normalized array data to the corresponding yeast intergenic sequences, eliciting motifs of up to length 8 and containing up to 2 IUPAC degenerate symbols. In order to verify the correctness of our predictions, we compared these results to a benchmarking set consisting of 65 high confidence motif logos assembled from the predictions of six separate motif finding algorithms [1]. For 47 of 65 benchmarks fREDUCE produced an IUPAC motif that was identical to the annotated motif, including correct degeneracies (Table 1). In comparison, we ran AlignACE [22,23] on the same 65 


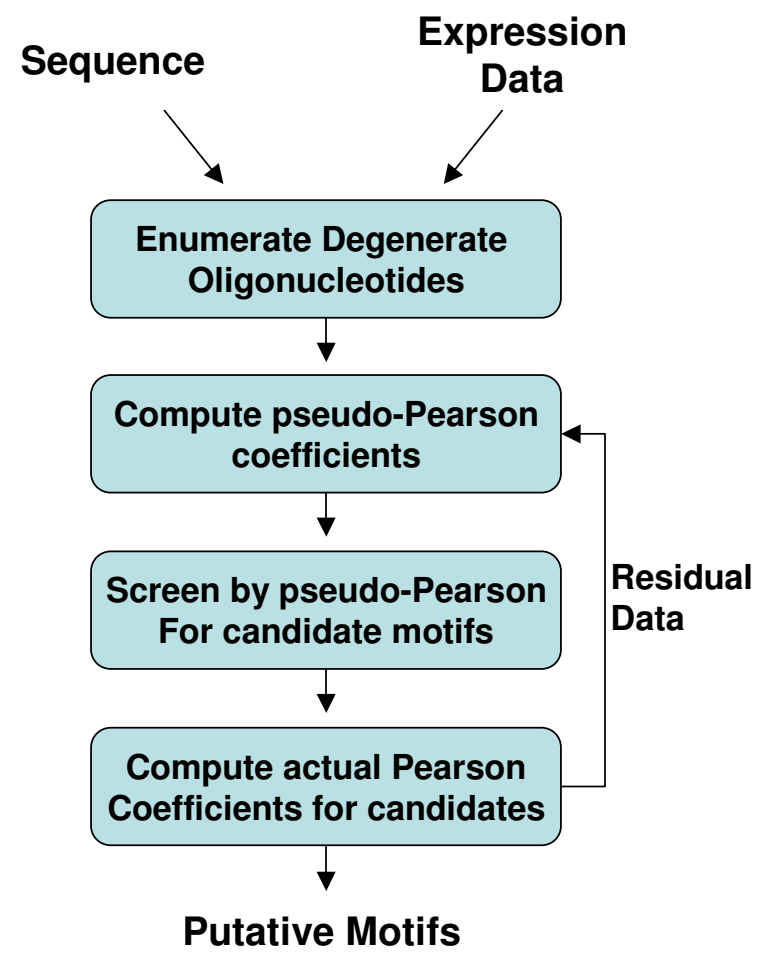

Figure I

The fREDUCE algorithm. A set of possible IUPAC strings are generated from the input sequence. For each IUPAC string, we compute a pseudo-Pearson coefficient, which is an estimate and upper bound on the true Pearson coefficient. After the vast majority of motifs are filtered out using the pseudo-Pearson value, we then compute true Pearson coefficients for the remaining motifs and select the top motif. The residual expression value is then used to iteratively derive subsequent motifs.

ChIP-chip experiments. Using the same filtering and comparison criteria, we found that AlignACE detected the annotated motif for only 36 of 65 regulators. We also compared the performance of fREDUCE with those of the other 5 motif finding algorithms used to assemble the benchmark motifs (Figure 2). Even though the benchmark motifs are likely to be biased toward the six programs from which they were originally found, fREDUCE still stood out as having the best individual performance.

We also examined the performance of fREDUCE on 38 regulators for which Harbison et. al. detected motifs with lower confidence [see Supplementary Tables]. Noting that many of these 38 predicted motifs could contain inaccuracies, fREDUCE matched 7 of these predictions while alignACE matched 3.

\section{Comparision to the original REDUCE and to MatrixREDUCE}

To assess the ability of fREDUCE to correctly capture motif degeneracies, we systematically compared the predictions made by fREDUCE to those made by its predecessor REDUCE on the subset of benchmark motifs containing significant degeneracy. Of 15 degenerate benchmark motifs, fREDUCE assigned IUPAC degenerate symbols identically to the benchmark in 11 cases (Figure 3 ). In the 4 remaining cases (HAP1, MSN2, STB5 and SUM1) fREDUCE made a prediction which is consistent with the benchmark motif while having a different degeneracy (e.g. CGGkGwTA vs. CGGwsTTA for STB5). In all of these cases, fREDUCE assigns the degenerate motif a more significant $\mathrm{p}$-value than the corresponding non-degenerate motif. We note that in some cases motif degeneracies can be detected by the original REDUCE as separate motif predictions. This is especially true for regulators with strong signal (AFT2, CIN5, FHL1, GCN4, SFP1 and YAP7). However, in 5 cases degeneracies successfully predicted by fREDUCE were not detectable at all by REDUCE (CAD1, PHO4, SNT2, TEC1 and YAP1). This is typically characteristic of regulators with weaker signal.

We also compared the performance of fREDUCE to MatrixREDUCE, a recently introduced REDUCE-variant that refines motifs elicited by REDUCE into Position Specific Affinity Matrices (PSAM) [24,25]. MatrixREDUCE matched 43 of the 65 benchmarks as well as 6 of 38 motifs in the lower confidence set [see Additional file 1]. In the high confidence set, six predictions were specific to fREDUCE (HAP4, HSF1, INO4, LEU3, NFG1 and THI2) while two were specific to MatrixREDUCE (MCM1, SIP4). Specific predictions from the lower confidence set included ROX1, SWI5, UME1 for fREDUCE and PUT3, RLM1 for MatrixREDUCE. Overall, fREDUCE has a slightly stronger joint performance with 9 uniquely correct predictions from the two sets versus MatrixREDUCE's 4. In the former cases, MatrixREDUCE did not seem to begin with the correct seed, suggesting that an enumeration strategy is beneficial for some regulators. In the latter cases, fREDUCE does not find the correct motif because the long and fuzzy nature of these motifs makes them too costly for enumeration. We note that some of these differences are dependent on run parameters; with the parameters we have used MatrixREDUCE took an order of magnitude longer to run on average than fREDUCE (data not shown).

\section{Prediction of novel motifs from yeast ChIP-chip}

Next we looked to see whether fREDUCE was capable of detecting novel motifs for transcription factors with uncharacterized specificities. Of the remaining transcription factors in the ChIP-chip study with no benchmark logo, we found 24 cases where fREDUCE made nontrivial 
Table I: fREDUCE motif predictions from yeast ChIP-chip

\begin{tabular}{|c|c|c|c|c|c|c|}
\hline Factor & Known Site & Condition & Motif & p-value & fREDUCE match? & AlignACE Match? \\
\hline ABFI & rTCAyt....Acg & YPD & rTGATm & 22.4 & $\sqrt{ }$ & $\sqrt{ }$ \\
\hline ACE2 & tGCTGGT & YPD & kGCTGGy & 6.2 & $\sqrt{ }$ & \\
\hline AFT2 & GGGTGy & $\mathrm{H} 2 \mathrm{O} 2 \mathrm{Lo}$ & rGGTGy & 91.5 & $\sqrt{ }$ & $\sqrt{ }$ \\
\hline AZFI & YwTTkcKkTyyckgykky & YPD & mTTTTw & 14.8 & & \\
\hline BAS I & TGACTC & YPD & TGACTCCG & 37.2 & $\sqrt{ }$ & $\sqrt{ }$ \\
\hline CADI & mTTAsTmAkC & YPD & GmTTAsTA & 4.2 & $\sqrt{ }$ & $\sqrt{ }$ \\
\hline CBF I & tCACGTG & YPD & CACGTG & 90.7 & $\sqrt{ }$ & $\sqrt{ }$ \\
\hline CIN5 & TTAygTAA & YPD & TTAyrTAA & 59.4 & $\sqrt{ }$ & $\sqrt{ }$ \\
\hline DAL82 & GATAAGa & RAPA & GATAAG & 9.4 & $\sqrt{ }$ & \\
\hline DIG I & TgAAAca & YPD & TGAAACA & 18 & $\sqrt{ }$ & \\
\hline FHLI & rTGTayGGrtg & YPD & GTAyGGrT & $14 \mid .2$ & $\sqrt{ }$ & $\sqrt{ }$ \\
\hline FKHI & tTgTTTac & YPD & yTGTTkAC & 28.8 & $\sqrt{ }$ & \\
\hline FKH2 & aaa.GTAAACAa & YPD & GTAAACA & 23.7 & $\sqrt{ }$ & $\sqrt{ }$ \\
\hline GAL4 & CGG............c & YPD & TTCGGAGC & 4.9 & & $\sqrt{ }$ \\
\hline GATI & aGATAAG & RAPA & GATAAG & 13.3 & $\sqrt{ }$ & \\
\hline GCN4 & TGAsTCa & YPD & rTGAsTCA & 166.7 & $\sqrt{ }$ & $\sqrt{ }$ \\
\hline GLN3 & GATAAGa.a & RAPA & GATAAG & 38.2 & $\sqrt{ }$ & \\
\hline HAPI & GGmraTA.CGs & YPD & kTTATCGG & 60.3 & $\sqrt{ }$ & $\sqrt{ }$ \\
\hline HAP4 & g. CcAAtcA & YPD & CCAATsAr & 21.7 & $\sqrt{ }$ & $\sqrt{ }$ \\
\hline HSF I & TTСуа.....TTC & $\mathrm{H} 2 \mathrm{O} 2 \mathrm{Hi}$ & TTCyrGAA & 109.5 & $\sqrt{ }$ & $\sqrt{ }$ \\
\hline IMEI & & $\mathrm{H} 2 \mathrm{O} 2 \mathrm{Hi}$ & & & & \\
\hline INO2 & CAcaTGc & YPD & kCACATGC & 12.8 & $\sqrt{ }$ & \\
\hline INO4 & CATGTGaaaa & YPD & CAyrTG & 89.2 & $\sqrt{ }$ & $\sqrt{ }$ \\
\hline LEU3 & cCGgtacCGG & YPD & CGGkACCG & 10.8 & $\sqrt{ }$ & $\sqrt{ }$ \\
\hline MBPI & rACGCGt & YPD & ACGCGT & 126.9 & $\sqrt{ }$ & $\sqrt{ }$ \\
\hline MCMI & tttCC.rAt..gg & Alpha & уТТССТАA & 5.7 & & $\sqrt{ }$ \\
\hline MET4 & RMmAwsTGKSgyGsc & SM & CrCGyG & 14.8 & & \\
\hline MSN2 & mAGGGGsgg & $\mathrm{H} 2 \mathrm{O} 2 \mathrm{Hi}$ & rGGGGy & 20.8 & $\sqrt{ }$ & \\
\hline NDDI & tt.CC.rAw..GG & YPD & CTCGAGGC & 12.3 & & $\sqrt{ }$ \\
\hline NRG I & $\mathrm{GGaCCCT}$ & YPD & AGGGTCs & 11.3 & $\sqrt{ }$ & $\sqrt{ }$ \\
\hline PDRI & ccGCCgRAwra & YPD & CCrwACAT & 11.4 & & \\
\hline PHDI & sc.GC.gg & YPD & mTGCAk & 21.1 & & $\sqrt{ }$ \\
\hline PHO2 & SGTGCGsygyG & $\mathrm{Pi}-$ & & & & \\
\hline $\mathrm{PHO4}$ & CACGTGs & $\mathrm{Pi}-$ & sCACGTGs & 14.1 & $\sqrt{ }$ & \\
\hline RAPI & tGyayGGrtg & SM & GyrTGGGT & 57.1 & $\sqrt{ }$ & $\sqrt{ }$ \\
\hline RCSI & ggGTGca.t & $\mathrm{H} 2 \mathrm{O} 2 \mathrm{LO}$ & GGGTGCA & 43.6 & $\sqrt{ }$ & $\sqrt{ }$ \\
\hline RDSI & kCGGCCGa & $\mathrm{H} 2 \mathrm{O} 2 \mathrm{Hi}$ & TCCGCGG & 35.6 & $\sqrt{ }$ & \\
\hline REB I & CGGGTAA & YPD & CGGGTAAy & 136.7 & $\sqrt{ }$ & $\sqrt{ }$ \\
\hline RFXI & TTgccATggCAAC & YPD & GTCGTCCG & 3.2 & & $\sqrt{ }$ \\
\hline RLRI & ATTTTCttCwTt & YPD & & & & \\
\hline RPN4 & TTTGCCACC & H2O2Lo & TyGCCACC & 109.8 & $\sqrt{ }$ & $\sqrt{ }$ \\
\hline SFPI & ayCcrtACay & SM & yCCrTACA & 31.6 & $\sqrt{ }$ & $\sqrt{ }$ \\
\hline SIG I & ArGmAwCrAmAA & $\mathrm{H} 2 \mathrm{O} 2 \mathrm{Hi}$ & & & & \\
\hline SIP4 & CGG.y.AATGGrr & SM & CTCGGCCC & 58.4 & & \\
\hline SKN7 & G.C..GsCs & H2O2Lo & GsCyGGCC & 37.7 & $\sqrt{ }$ & \\
\hline SNT2 & yGGCGCTAyca & YPD & GrTAGCGC & 96.1 & $\sqrt{ }$ & $\sqrt{ }$ \\
\hline SOK2 & tGCAg..a & BUTI4 & GGTrCAGA & 5.6 & & \\
\hline SPT2 & ymtGTmTytAw & YPD & TkyATA & 6.2 & & \\
\hline SPT23 & rAAATsaA & YPD & wTkAAA & 25.1 & & \\
\hline STB I & rracGCsAaa & YPD & wCGCGT & 4 & $\sqrt{ }$ & \\
\hline STB4 & TCGg..CGA & YPD & CGGryCGA & 7.1 & $\sqrt{ }$ & $\sqrt{ }$ \\
\hline STB5 & CGGwstTAta & YPD & CGGkGwTA & 24 & $\sqrt{ }$ & \\
\hline STEI 2 & $\operatorname{tg} A A A C a$ & YPD & TGAAACA & 38.9 & $\sqrt{ }$ & $\sqrt{ }$ \\
\hline SUMI & gyGwCAswaaw & YPD & GyGTCAs & 25.0 & $\sqrt{ }$ & $\sqrt{ }$ \\
\hline SUTI & gcsGsg..sG & YPD & wCkCCG & 49.8 & & \\
\hline SWI4 & raCgCsAAA & YPD & CGCsAAAA & 12.6 & $\sqrt{ }$ & $\sqrt{ }$ \\
\hline SWI6 & tttcGCGt & YPD & TTTCsk & 11.6 & $\sqrt{ }$ & \\
\hline TECI & rrGAATG & YPD & rrGAATGT & 22.4 & $\sqrt{ }$ & \\
\hline THI2 & gmAAcy.twAgA & Thi- & GGAAACyS & 4.5 & $\sqrt{ }$ & \\
\hline
\end{tabular}


Table I: fREDUCE motif predictions from yeast ChIP-chip (Continued)

\begin{tabular}{ccccccc}
\hline TYE7 & tCACGTGAy & YPD & TCACGTGr & 70.8 & $\sqrt{ }$ & $\sqrt{ }$ \\
UME6 & taGCCGCCsa & YPD & GCsGCy & 154.3 & $\sqrt{ }$ & $\sqrt{ }$ \\
YAPI & TTaGTmAGc & YPD & mTkACTAA & 13.6 & $\sqrt{ }$ & $\sqrt{ }$ \\
YAP7 & mTkAsTmAk & H2O2Hi & mTTAsTAA & 121.9 & $\sqrt{ }$ & $\sqrt{ }$ \\
YDR026c & ttTACCCGGm & YPD & CCGGGTAA & 23.2 & $\sqrt{ }$ & $\sqrt{ }$ \\
ZAPI & ACCCTmAAGGTyrT & YPD & wAyATT & 16.5 & &
\end{tabular}

fREDUCE predictions from 65 yeast ChIP-chip experiments of Harbison et. al. Check marks $(\sqrt{ })$ indicate that fREDUCE matched the IUPAC string corresponding to the benchmark logo. The results of a similar analysis with AlignACE is given in the right column.

(not repetitive poly-dA/dT sequences) motif predictions with p-values under $10^{-3}$ (Table 2). In all of these cases, there has been little to no experimental information available regarding the specificity, and existing computation methods have yielded little additional insight. Nevertheless, in a few cases we found evidence in the literature which supports the novel motif predictions we have made with fREDUCE. For example, the binding site of ARO80, a regulator of the aromatic amino acid structural genes, has been characterized in two genes as being tandem repeats of the sequences TAACCG and TTGCCG [26]. From the ChIP-chip data, fREDUCE elicited the motif GATAACCG with high significance $\left(\mathrm{p}=10^{-41}\right)$ as well as the degenerate motif $\mathrm{T}(\mathrm{A} / \mathrm{G}) \mathrm{CCG}(\mathrm{A} / \mathrm{C})\left(\mathrm{p}=10^{-5.6}\right)$, which is similar to both of the characterized repeat elements and reflects their degeneracies. We also considered the regulator MTH1, which negatively regulates the glucose sensing signal transduction pathway by interacting with the transcriptional repressor Rgt1p [27]. Although it is unknown

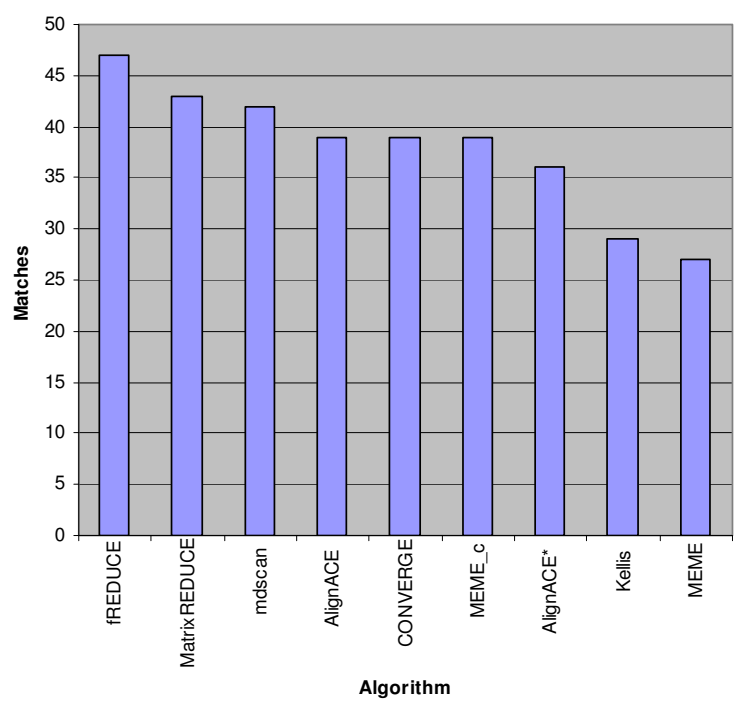

Figure 2

Comparison of fREDUCE to six other algorithms on 65 yeast ChIP-chip benchmarks. AlignACE* indicates results of running AlignACE from scratch, while the performance of other methods were compiled from the Harbison et. al supporting website. whether Mth1p has intrinsic DNA sequence specificity, Rgt1p has been shown to have the specificity CGGANNA [28]. fREDUCE found the matching motif GGAGRA ( $\mathrm{p}=$ $10^{-3.57}$ ), which is compatible with the notion that Mth1p binds to DNA in association with Rgt1p.

\section{Motif Elicitation in Human Hepatocytes}

In higher eukaryotes, motifs tend to be more degenerate and dispersed among longer intergenic regions. A common benchmark set used to evaluate the performance of computational algorithms in higher eukaryotes is the liver specific dataset [29]. Krivan et. al compiled a set of experimentally defined regulatory elements upstream of genes that were expressed exclusively in liver or in a small number of tissues including liver. From this set of genes, they found that hepatocyte-specific gene expression is mainly regulated by a small set of transcription factors (TFs), including HNF-1, HNF-3, HNF-4, and C/EBP. HNF1, HNF-4, and C/EBP are known to be transcriptional activators based on TRANSFAC [30] annotation.

We ran fREDUCE on human adult hepatocyte expression data to capture binding sites of liver-specific transcription factors. fREDUCE captured both the forward and reverse complement of the HNF-4 binding site as well as two key degeneracies in the motif core as published in Krivan et. al. (Figure 4). HNF-4 is known to be linked to gene expression in mature liver [29], which is consistent with the expression data set used in our analysis. In contrast, REDUCE was not able to capture the known binding sites, which is most likely due to the degeneracy involved in the known consensus. These results show the potential of using fREDUCE to identify regulatory elements in higher eukaryotes, including human.

\section{Discussion}

Despite the availability of powerful techniques such as ChIP-chip, the binding specificities of many transcription factors remain uncharacterized. This can be due to several reasons, including 1 ) regulators that have few genomic targets 2) regulators which interact weakly or indirectly with their targets and 3) regulators which bind to their maximal set of targets only under very specific environmental cues, which may be hard to find experimentally. fREDUCE offers increased sensitivity in these cases 


\begin{tabular}{|c|c|c|c|c|}
\hline TF & $\begin{array}{c}\text { REDUCE } \\
(\mathrm{p}-\mathrm{value})\end{array}$ & $\begin{array}{c}\text { fREDUCE } \\
(\mathrm{p}-\mathrm{value})\end{array}$ & Benchmark Logo & $\begin{array}{c}\text { Benchmark } \\
\text { Motif }\end{array}$ \\
\hline AFT2 & $\begin{array}{l}\text { GGGTGC (61.8) } \\
\text { GGGTGT }(31.6)\end{array}$ & GGGTGy (91.5) & & GGGTGY \\
\hline CAD1 & $\begin{array}{c}\text { ATTAGTA (2.9) } \\
-\end{array}$ & GmTTAsTA (4.2) & & mTTAsTmAkC \\
\hline CIN5 & $\begin{array}{l}\text { TATGTAA }(17.8) \\
\text { TACGTAA }(15.6)\end{array}$ & $\begin{array}{l}\text { TTAyrTAA } \\
(59.4)\end{array}$ & & TTAYGTAA \\
\hline FHL1 & $\begin{array}{l}\text { TGTACGG }(59.4) \\
\text { GTATGGG }(30.5)\end{array}$ & $\begin{array}{l}\text { GTAYGGrT } \\
(159.7)\end{array}$ & & rTGTayGGrt \\
\hline GCN4 & $\begin{array}{r}\text { TGACTCA }(103.3) \\
\text { GAGTCAT }(36.4)\end{array}$ & $\begin{array}{l}\text { rTGAsTCA } \\
(166.7)\end{array}$ & & TGAsTCA \\
\hline HAP1 & $\begin{array}{c}\text { TATCGG }(38.8) \\
-\end{array}$ & $\begin{array}{l}\text { RTTATCGG } \\
(60.3)\end{array}$ & & GGmraTA. CGs \\
\hline MSN2 & $\begin{array}{c}\text { AAGGGG }(8.6) \\
-\end{array}$ & rGGGGy $(20.8)$ & & mAGGGGsgg \\
\hline $\mathrm{PHO} 4$ & $\begin{array}{c}\text { CACGTGC (6.4) } \\
- \\
\end{array}$ & $\begin{array}{c}\text { sCACGTGs } \\
(14.1)\end{array}$ & & CACGTGS \\
\hline SFP1 & $\begin{array}{l}\text { CCGTACA }(12.2) \\
\text { CCCATAC }(10.4)\end{array}$ & $\begin{array}{l}\text { YCCrTACA } \\
(31.6)\end{array}$ & & ayCcrtACay \\
\hline SNT2 & $\begin{array}{r}\text { GGCGCTA }(49.7) \\
\text { CGCTATC }(7.0)\end{array}$ & $\begin{array}{c}\text { GCGCTAYC } \\
(96.1)\end{array}$ & & YGGCGCTAYca \\
\hline STB5 & $\begin{array}{c}\text { CGGTGTT ( } 7.0) \\
-\end{array}$ & $\begin{array}{c}\text { CGGkGwTA } \\
(24.0)\end{array}$ & & CGGwstTAta \\
\hline SUM1 & $\begin{array}{l}\text { TGTCAC }(11.4) \\
\text { TGACAC }(8.9)\end{array}$ & $\begin{array}{l}\text { GWCAGTAA } \\
(25.0)\end{array}$ & & gyGwCAswaa \\
\hline TEC1 & $\begin{array}{c}\text { AGAATG }(13.0) \\
-\end{array}$ & $\begin{array}{l}\text { IrGAATGT } \\
(22.4)\end{array}$ & & rrGAATG \\
\hline YAP1 & $\begin{array}{c}\text { ATTAGT }(10.9) \\
-\end{array}$ & $\begin{array}{l}\text { TTAGTmAk } \\
(13.6)\end{array}$ & & TTaGTmAGc \\
\hline YAP7 & $\begin{array}{l}\text { TTACTAA }(50.1) \\
\text { TTAGTAA }(41.7) \\
\text { TGACTAA }(15.9)\end{array}$ & $\begin{array}{c}\text { TTAsTAAk } \\
(118.6)\end{array}$ & & mTkAsTmAk \\
\hline
\end{tabular}

\section{Figure 3}

fREDUCE predictions in comparison to non-degenerate predictions made by REDUCE. Benchmark logos and their corresponding motifs are shown for reference. P-values are shown as - $\log _{10}$ values.

because it 1) uses the entire array data set for correlation and 2) searches all possible degeneracies. While fREDUCE is in some respects similar to motif regressor [14] and matrixREDUCE, a key distinction is that fREDUCE detects degenerate motifs de novo by exhaustive enumeration. In contrast, matrixREDUCE refines degeneracies from nondegenerate seeds and motif regressor selects among candidate matrices using correlation with expression. Thus, fREDUCE may be advantageous when motifs are difficult 
Table 2: fREDUCEpredictions for regulators with poorly characterized specificities

\begin{tabular}{|c|c|c|c|}
\hline Regulator & Predicted Site & P-value & Motif from Literature Search \\
\hline ARG80 & TTYTCY & 34.3 & CYNYYAANKRMAR \\
\hline ARO80 & TRCCGM & 5.6 & TWRCCG \\
\hline ASKIO & AYTTKA & 9.1 & \\
\hline CST6 & TYAAWA & 7.0 & \\
\hline DATI & WTTSAA & 16.7 & \\
\hline ECM22 & GCRSCC & 16.2 & TCGTATA \\
\hline EDSI & TWTTSA & 8.4 & \\
\hline FAP7 & WTRAAG & 11.3 & \\
\hline GAT3 & CCTSGGC & 15.2 & \\
\hline GCR2 & TTCAWW & 5.0 & СТTCC \\
\hline HAL9 & WTTRAA & 14.7 & \\
\hline HIR3 & WTTRAA & 22.0 & ACGCTAAA \\
\hline IME4 & YACACAC & 17.8 & \\
\hline MALI3 & CCASSG & 11.6 & \\
\hline MAL33 & GCRCAS & 13.8 & \\
\hline METI 8 & WTTCAA & 8.2 & \\
\hline MGAI & TTTRAY & 5.9 & \\
\hline MSNI & MMCCCA & 3.8 & \\
\hline MTHI & GGAGRA & 3.4 & CGGANNA * \\
\hline OAFI & CGCASY & 4.9 & CGGNNNTNAN $_{9-12}$ CCG \\
\hline RGMI & CSGSCC & 27.1 & \\
\hline RTG I & ATYTRA & 10.3 & \\
\hline SIP3 & WTCAAW & 7.6 & \\
\hline SMKI & WTGWAG & 3.9 & \\
\hline STB2 & CAAGGYC & 3.1 & \\
\hline STB6 & TATSAW & 5.6 & \\
\hline STP4 & AARMTT & 24.1 & \\
\hline TOS8 & RCACMC & 20.7 & \\
\hline UPC2 & MATSAA & 4.5 & \\
\hline WARI & TYAAGW & 6.6 & \\
\hline YBR239c & WATAYT & 16.8 & \\
\hline YDR049W & AWTGAW & 3.5 & \\
\hline YER05 I W & AKYACT & 3.9 & \\
\hline YERI30C & CAARTW & 3.1 & \\
\hline YFL052w & WTCAAK & 3.6 & \\
\hline YGR067C & TTYAAW & 4.6 & \\
\hline YKR064W & WGTTRA & 6.3 & \\
\hline YLR278C & KTTMAA & 7.2 & \\
\hline YML08IW & WCAAMT & 3.7 & \\
\hline YNR063W & TCAARTA & 2.4 & \\
\hline YPRI96W & WTCAAW & 10.3 & \\
\hline
\end{tabular}

We searched the literature for evidence supporting our motif predictions and the matching examples are highlighted. *The annotated motifs for Rgtlp.

to detect in a non-degenerate form or are missed in the candidate set.

By comparison to 65 benchmark logos in yeast, we see that fREDUCE is comparable to or greater in detection power versus algorithms like AlignACE for strong motifs that are relatively easy to detect. Even in these cases, fREDUCE outperforms the original REDUCE algorithm by accurately predicting known degeneracies. The most advantageous use of fREDUCE, however, is for the detection of weak motifs which may lie at the border of detection. It is difficult to verify the correctness of many of the motifs elicited in these cases because of their poor characterization. Nevertheless, we have found two cases where fREDUCE was sensitive to subtle signals: ARO80, for which sites are highly degenerate, and MTH1, which may have a weak signal due an indirect interaction with DNA. We have also shown that fREDUCE is capable of capturing the HNF-4 binding site in hepatocytes, demonstrating that this algorithm is generally applicable to the detection of degenerate motifs in mammalian cells. 


\begin{tabular}{|c|c|c|c|c|}
\hline TF & $\begin{array}{c}\text { REDUCE } \\
(\mathrm{p}-\mathrm{value})\end{array}$ & $\begin{array}{c}\text { fREDUCE } \\
(\mathrm{p}-\mathrm{value})\end{array}$ & Benchmark Logo & $\begin{array}{c}\text { Benchmark } \\
\text { Motif }\end{array}$ \\
\hline $\mathrm{HNF}-4$ & - & GRMCTTTG (7.4) & TG & TGrmCTTTG \\
\hline
\end{tabular}

\section{Figure 4}

fREDUCE elicitation of the HNF-4 binding site from human hepatocyte expression data.

\section{Conclusion}

We have presented the motif prediction algorithm fREDUCE, a refined variation of REDUCE specialized for the detection of degenerate motifs. The two primary strengths of fREDUCE are 1) it maximizes data utilization by fitting all expression data and 2) it searches motif degeneracies in a comprehensive and unbiased way. We have shown that fREDUCE is an improvement upon the existing REDUCE algorithm for degenerate binding profiles and that it can outperform existing motif finding methods on yeast ChIP-chip benchmarks. Furthermore, fREDUCE is able to detect degenerate signals in yeast and human. Thus, fREDUCE should be a valuable computation tool for the detection of subtle motifs.

\section{Methods}

\section{Algorithm}

The pearson correlation between expression values and counts of a possibly degenerate motif $D$ is given by:

$$
P(D)=\frac{\sum_{i=1}^{G}\left(E_{i}-\bar{E}\right)\left(n_{i}^{D}-\overline{n^{D}}\right)}{\sqrt{\sum_{i=1}^{G}\left(E_{i}^{2}-\bar{E}^{2}\right)} \cdot \sqrt{\sum_{i=1}^{G}\left(n_{i}^{D} n_{i}^{D}-\bar{n}^{2}\right)}}
$$

Where $i$ is an index over genes, $E_{i}$ is the expression of gene $i, n_{i} D$ is the number of motif counts matching $D$ in sequence $i, \bar{n}$ is the average of $n_{i}^{D}$ over all genes and $G$ is the total number of genes. Let $g_{i}$ be the normalized gene expression: $g_{i}=\frac{E_{i}-\bar{E}}{\sqrt{\sum_{i=1}^{G}\left(E_{i}^{2}-\bar{E}^{2}\right)}}$, so that $\sum_{i=1}^{G} g_{i}=0$ and $\sum_{i=1}^{G} g_{i}^{2}=1$. Then the Pearson coefficient reduces to:

$$
P(D)=\frac{\sum_{i=1}^{G} g_{i} n_{i}^{D}}{\sqrt{\sum_{i=1}^{G}\left(n_{i}^{D} n_{i}^{D}-\bar{n}^{2}\right)}}
$$

Since $n_{i}^{D}=\sum_{S} n_{i}^{S}$, where the sum is over all non-degenerate nucleotide motifs $S$ that match $D$, we can pre-compute and store a table of $\sum_{i=1}^{G} g_{i} n_{i}^{S}$ for all $S$ and readily construct the numerator of $P(D)$ for any $D$. However, the denominator is not linear in $n_{i} D$ and cannot be expressed as a sum over $S$. Nevertheless we can compute a pseudo-Pearson coefficient:

$$
\tilde{P}(D)=\frac{\sum_{i=1}^{G} g_{i} n_{i}^{D}}{\sqrt{\tilde{n}^{2}-G \bar{n}^{2}}}
$$

where $\tilde{n}^{2}=\sum_{S} \sum_{i=1}^{G} n_{i}^{S} n_{i}^{S}$ can be constructed as a sum over $S$.

Since

$$
\sum_{i=1}^{G} n_{i}^{D} n_{i}^{D}=\sum_{i=1}^{G}\left(\sum_{S_{1}} n_{i}^{S_{1}}\right)\left(\sum_{S_{2}} n_{i}^{S_{2}}\right)=\sum_{S_{1}} \sum_{S_{2}} \sum_{i=1}^{G} n_{i}^{S_{1}} n_{i}^{S_{2}} \geq \tilde{n}^{2},
$$

we have $|P(D)| \leq|\tilde{P}(D)|$. Hence the magnitude of pseudo-Pearson coefficient is an upper bound for the magnitude of the actual Pearson coefficient, allowing rapid screening of all degenerate motifs. Actual Pearson values can then be computed for a small subset of motifs with pseudo-Pearson values above a given threshold. This scheme is effective except for motifs where $\tilde{n}^{2}<G \bar{n}^{2}$, in which case the Pearson coefficient must be computed 
directly. Thus, fREDUCE will give a computational advantage as long as the average motif count $\bar{n}$ is less than one.

Specifically, fREDUCE uses the following procedure:

(1) For each oligonucleotide string $S$ of length $L$ that appears in the sequence, we pre-compute the quantities $p_{d}^{S}=\sum_{i=1}^{G} g_{i} n_{i}^{S}, \bar{n}^{S}=\frac{1}{G} \sum_{i=1}^{G} n_{i}^{S}$ and $\bar{n}^{2} S=\sum_{i=1}^{G} n_{i}^{S} n_{i}^{S}$

(2) We generate a list of all possible nucleotides containing up to $l$ degeneracies matching the set of $S$.

(3) We rapidly compute corresponding quantities for all degenerate strings $D$ matching $S$ : $p_{d}=\sum_{i=1}^{G} g_{i} n_{i}^{D}=\sum_{S} p_{d}{ }^{S}, \bar{n}=\frac{1}{G} \sum_{i=1}^{G} n_{i}^{D}=\sum_{S} \bar{n}^{S}$, and $\tilde{n}^{2}=\sum_{S} \sum_{i=1}^{G} n_{i}^{S} n_{i}^{S}=\sum_{S} \overline{n^{2}}$ and use them to construct the pseudo-Pearson coefficient $p_{D} / \sqrt{\tilde{n}^{2}-G \bar{n}^{2}}$. We save only those motifs whose pseudo-Pearson coefficients exceed a threshold corresponding to the p-value cutoff for its motif class. For the motifs whose pseudo-Pearson coefficients cannot be calculated directly (because $\tilde{n}^{2} \leq G \bar{n}^{2}$ ), we compute the true Pearson.

(4) We sort the remaining motifs in decreasing order of the magnitudes of their pseudo-Pearson and compute true Pearson coefficients in this order. We stop computing when the magnitude of the pseudo-Pearson value of the current motif in the list falls below the magnitude of the true Pearson coefficient of the top motif.

(5) Finally, we compute the residual gene expression $\tilde{g}_{i}=g_{i}-P(D) n_{i}^{D}$, that is, the expression data after the effect of motif $D$ has been taken into account. After a renormalization, the residual is used to carry out subsequent rounds of motif finding.

To estimate the statistical significance of motifs, we note that since $|P(D)|<<1$, its distribution is well approximated by a Normal distribution. We convert $\mathrm{P}(\mathrm{D})$ into a $\mathrm{z}$-score:

$$
Z(D)=P(D) \sqrt{\frac{G-2}{1-P(D)^{2}}}
$$

This z-score is used to derive the p-value [15]:

$$
\text { pvalue }=\frac{2}{\sqrt{2 \pi}} \int_{Z(D)}^{\infty} e^{\frac{-t^{2}}{2}} d t
$$

To correct for multiple testing, we first apply a Bonferroni correction factor of $\left(\begin{array}{c}L \\ m\end{array}\right) D^{m} 4^{L-m}$ to each motif of length $L$ containing $m$ IUPAC symbols. This factor corresponds to the total number of motifs in the class of $L$ and $m$, where $D=11$ or 15 depending on whether 3 -fold IUPAC symbols are included. We then apply a second correction factor for the total number of motif classes examined for a particular run. For example, with the settings $(\mathrm{L}=7$ and $\mathrm{m}=1$ ) we would examine all motifs in the classes $(6,0)$, $(6,1),(7,0)$ and $(7,1)$ giving a second correction factor of 4 for each motif (we require a minimum motif length of 6 ). This weighted method of correction has the advantage of accounting for the fact that motif classes with larger values of $L$ and $m$ tend to give higher numbers of false positives.

\section{fREDUCE performance testing}

We ran fREDUCE on the REB1_YPD ChIP-chip data from Harbison et. al. for varying $L$ and $m$ on an $2.40 \mathrm{GHz}$ Intel Xeon processor [see Additional file 2]. In all runs, the known Reb1p binding site CGGGTAA or close variants appeared as the top motif (data not shown).

\section{Motif Detection from Yeast ChIP-chip}

We applied fREDUCE to 354 yeast ChIP-chip experiments involving 203 known and putative transcription factors [1]. Each experiment was analyzed with fREDUCE using the corresponding set of yeast intergenic sequences, searching all motifs up to length 8 containing up to 2 twofold IUPAC degenerate symbols. We filtered the set of motifs found for each fREDUCE run by three criteria. First, since yeast intergenic sequences have relatively low $\mathrm{G} / \mathrm{C}$ content, we eliminated motifs which only contained the letters $\mathrm{A} / \mathrm{T} / \mathrm{W}$ as such motifs tend to have inflated correlation coefficients. From the remaining list of motifs, we chose the top three most significant motifs for further comparison. Accounting for the fact that we are eliciting motifs from several hundred experiments, we also discarded motifs with corrected p-values less significant than $10^{-2}$. If the given transcription factor was associated with ChIP-chip data under multiple environmental conditions, then filtered motifs from all conditions were combined and the top three chosen. The final motifs for each transcription factor were compared to reference motifs predicted by Harbinson et. al. based on a composite of several motif finding algorithms [1]. There were a total of 102 reference motifs from the authors' website [31], 65 of which 
were considered high confidence. Each reference motif was compared to their corresponding fREDUCE predictions using a sliding window string comparison. Predicted motifs are considered a match if there is at least one window where all IUPAC characters are consistent between both strings. Motif predictions made for transcription factors with no reference motifs were compared to literature.

\section{Comparison to non-degenerate REDUCE}

From the 65 high confidence benchmarks, we selected cases where the annotated motif had at least one IUPAC character. In 15 of these cases, both fREDUCE and REDUCE made correct, if not correctly degenerate predictions. In 11 of these 15 cases fREDUCE made the correct IUPAC assignments. For each of these 11 cases, we considered whether the degeneracy can be assembled from nondegenerate motifs with $\mathrm{p}<0.01$ predicted by REDUCE.

\section{Comparison to other motif-finding algorithms}

We obtained the alignACE package and ran all ChIP-chip data with the default parameters using probes with p-values below 0.001 . The output alignment was converted into an IUPAC string using the method described by Cavener et. al. [32] and the resulting motifs were compared to reference motifs in the same way as the fREDUCE motif predictions. Details of alignACE motifs found and comparisons to alignACE motifs from Harbison et. al. are available in Supp. Table 1. We also obtained MatrixREDUCE [33] and ran all ChIP-chip data against the provided yeast sequence file Y5_600_Bst.fa. Default parameters were used except that we set max_motif $=10$ for consistency with our fREDUCE runs. For the other five algorithms, we tallied the total number of references to each algorithm from the list of matrices on Harbison et al. supporting website [34].

\section{Motif Detection from Human Liver Tissue}

158 custom made Affymetrix gene expression arrays for 79 different human tissues (2 replicates each) were obtained from Novartis in a publicly available database $[35,36]$. The arrays were normalized using gcrma $[37,38]$ and the probes were annotated using Ensembl gene annotation [39] for human build 35. To study adult liver specific gene expression, we first normalized expression values for each liver tissue replicate against the average expression of all other tissues (excluding the 2 liver tissue experiments) The expression value of each gene in liver tissue experiments is represented as the following $\mathrm{z}$-score:

$$
z^{n, g}=\frac{E^{n, g}{ }_{\text {liver }}-\mu_{\text {other }}^{g}}{\sigma_{\text {other }}^{g}}
$$

Where $n$ is the liver tissue experiment replicate number, $g$ is the index over genes, $E^{\text {ng }}{ }_{\text {liver }}$ is the expression value of gene $g$ in replicate $n, \mu_{\text {other }}$ is the mean expression value of gene $g$ in non-liver tissue experiments, and $\sigma_{\text {other }}$ is the standard deviation of gene $g$ in non-liver tissue experiments.

Human genomic sequences (build 35) were extracted 1000 bp upstream from the transcriptional start site (TSS) if known, or from the initiation codon, based on Ensembl v35 [40]. The repeat masked promoter sequences were mapped to corresponding $\mathrm{z}$-scores, which represent gene expression. This resulted in a final set of 11,710 paired $z-$ scores and promoter sequences for input into fREDUCE. We then ran fREDUCE on the $\mathrm{z}$-scores for each replicate of the liver tissue on the basis that a higher $\mathrm{z}$-score translates to higher expression in liver tissues compared to the other tissues. Two different sets of parameters were run on each replicate as follows: length 8 with 0 IUPAC symbols and length 8 with 2 IUPAC symbols.

\section{Availability and Requirements \\ - Project Name: fREDUCE}

- Project Home Page: http://genome3.ucsf.edu:8080/fre duce

- Operating system: Linux

\section{- Programming languages: $\mathrm{C}++$}

Source code and example usage are included in the release file fREDUCE-1.1.tar.gz [see Additional file 3].

\section{List of Abbreviations}

ChIP-on-chip: chromatin immunoprecipitation on microarray; REDUCE: regulatory element detection using correlation with expression; fREDUCE: fast regulatory element detection using correlation with expression.

\section{Competing interests}

The author(s) declares that there are no competing interests.

\section{Authors' contributions}

RW carried out the ChIP-chip calculations and drafted the manuscript. CC carried out the hepatocyte calculations and helped with the manuscript. JZ implemented the web interface. SL \& HL conceived of the algorithm. SL implemented the algorithm. HL supervised algorithm development and data analysis. All authors read and approved the final manuscript. 


\section{Additional material}

\author{
Additional file 1 \\ Supplementary Tables. Tables showing the details of motif comparisons of \\ fREDUCE against AlighACE and MatrixREDUCE are included here. \\ Click here for file \\ [http://www.biomedcentral.com/content/supplementary/1471- \\ 2105-8-399-S1.doc]

\section{Additional file 2} \\ Supplementary Figure 1. fREDUCE performance scaling as the motif \\ length and number of degeneracies allowed is varied. \\ Click here for file \\ [http://www.biomedcentral.com/content/supplementary/1471- \\ 2105-8-399-S2.ppt]

\section{Additional file 3} \\ fREDUCE-1.1.tar.gz. fREDUCE source \\ Click here for file \\ [http://www.biomedcentral.com/content/supplementary/1471- \\ 2105-8-399-S3.gz]
}

\section{Acknowledgements}

We would like to thank Jim Shima from the Conklin lab for providing the normalized and annotated Novartis human tissue dataset. This work is funded by predoctoral fellowships from the California Institute of Quantitative Biology (RW) and the National Science Foundation (CC). Funding for $\mathrm{HL}$ is provided by NIH (GM70808) and the David and Lucille Packard Fellowship.

\section{References}

I. Harbison CT, Gordon DB, Lee TI, Rinaldi NJ, Macisaac KD, Danford TW, Hannett NM, Tagne JB, Reynolds DB, Yoo J, et al.: Transcriptional regulatory code of a eukaryotic genome. Nature 2004, $431: 99-104$.

2. Lee TI, Rinaldi NJ, Robert F, Odom DT, Bar-Joseph Z, Gerber GK, Hannett NM, Harbison CT, Thompson CM, Simon I, et al.: Transcriptional regulatory networks in Saccharomyces cerevisiae. Science 2002, 298:799-804.

3. Ren B, Robert F, Wyrick JJ, Aparicio O, Jennings EG, Simon I, Zeitlinger J, Schreiber J, Hannett N, Kanin E, et al.: Genome-wide location and function of DNA binding proteins. Science 2000, 290:2306-2309.

4. Hughes TR, Marton MJ, Jones AR, Roberts C], Stoughton R, Armour $C D$, Bennett HA, Coffey E, Dai H, He YD, et al.: Functional discovery via a compendium of expression profiles. Cell 2000, 102:109-126.

5. Wang W, Cherry JM, Botstein D, Li H: A systematic approach to reconstructing transcription networks in Saccharomycescerevisiae. Proc Natl Acad Sci USA 2002, 99:16893-16898.

6. Bailey TL, Elkan C: Fitting a mixture model by expectation maximization to discover motifs in biopolymers. Proc Int Conf Intell Syst Mol Biol 1994, 2:28-36.

7. Lawrence CE, Altschul SF, Boguski MS, Liu JS, Neuwald AF, Wootton JC: Detecting subtle sequence signals: a Gibbs sampling strategy for multiple alignment. Science 1993, 262:208-214.

8. Liu X, Brutlag DL, Liu JS: BioProspector: discovering conserved DNA motifs in upstream regulatory regions of co-expressed genes. Pac Symp Biocomput 2001:127-138.

9. van Helden J, Andre B, Collado-Vides J: Extracting regulatory sites from the upstream region of yeast genes by computa- tional analysis of oligonucleotide frequencies. J Mol Biol 1998 , 281:827-842.

10. Wang T, Stormo GD: Combining phylogenetic data with coregulated genes to identify regulatory motifs. Bioinformatics 2003, 19:2369-2380.

II. Bussemaker $\mathrm{HJ}, \mathrm{Li} \mathrm{H}$, Siggia ED: Building a dictionary for genomes: identification of presumptive regulatory sites by statistical analysis. Proc Natl Acad Sci USA 2000, 97: 10096-10100.

12. Wang G, Zhang W: A steganalysis-based approach to comprehensive identification and characterization of functional regulatory elements. Genome Biol 2006, 7:R49.

13. Beer MA, Tavazoie S: Predicting gene expression from sequence. Cell 2004, I 17:185-198.

14. Conlon EM, Liu XS, Lieb JD, Liu JS: Integrating regulatory motif discovery and genome-wide expression analysis. Proc Natl Acad Sci USA 2003, 100:3339-3344.

15. Bussemaker HJ, Li H, Siggia ED: Regulatory element detection using correlation with expression. Nat Genet 200I, 27:167-I7I.

16. Kim Guisbert K, Duncan K, Li H, Guthrie C: Functional specificity of shuttling hnRNPs revealed by genome-wide analysis of their RNA binding profiles. RNA 2005, I I:383-393.

17. Klebes A, Sustar A, Kechris K, Li H, Schubiger G, Kornberg TB: Regulation of cellular plasticity in Drosophila imaginal disc cells by the Polycomb group, trithorax group and lama genes. Development 2005, 132:3753-3765.

18. Koerkamp MG, Rep M, Bussemaker HJ, Hardy GP, Mul A, Piekarska K, Szigyarto CA, De Mattos JM, Tabak HF: Dissection of transient oxidative stress response in Saccharomyces cerevisiae by using DNA microarrays. Mol Biol Cell 2002, 13:2783-2794.

19. Orian A, van Steensel B, Delrow J, Bussemaker HJ, Li L, Sawado T, Williams E, Loo LW, Cowley SM, Yost C, et al.: Genomic binding by the Drosophila Myc, Max, Mad/Mnt transcription factor network. Genes Dev 2003, 17:1101-11114.

20. van Steensel B, Delrow J, Bussemaker HJ: Genomewide analysis of Drosophila GAGA factor target genes reveals contextdependent DNA binding. Proc Natl Acad Sci USA 2003, 100:2580-2585.

21. Wang W, Cherry JM, Nochomovitz Y, Jolly E, Botstein D, Li H: Inference of combinatorial regulation in yeast transcriptional networks: a case study of sporulation. Proc Natl Acad Sci USA 2005, 102:1998-2003.

22. AlignACE Homepage [http://atlas.med.harvard.edu]

23. Roth FP, Hughes JD, Estep PW, Church GM: Finding DNA regulatory motifs within unaligned noncoding sequences clustered by whole-genome mRNA quantitation. Nat Biotechnol 1998, 16:939-945.

24. Foat BC, Houshmandi SS, Olivas WM, Bussemaker HJ: Profiling condition-specific, genome-wide regulation of mRNA stability in yeast. Proc Natl Acad Sci USA 2005, 102: I7675-17680.

25. Foat BC, Morozov AV, Bussemaker HJ: Statistical mechanical modeling of genome-wide transcription factor occupancy data by MatrixREDUCE. Bioinformatics 2006, 22:e I 4I-I49.

26. De Rijcke M, Seneca S, Punyammalee B, Glansdorff N, Crabeel M: Characterization of the DNA target site for the yeast ARGR regulatory complex, a sequence able to mediate repression or induction by arginine. Mol Cell Biol 1992, I 2:68-8I.

27. Lakshmanan J, Mosley AL, Ozcan S: Repression of transcription by Rgt I in the absence of glucose requires Std I and Mth I. Curr Genet 2003, 44:19-25.

28. Kim JH, Polish J, Johnston M: Specificity and regulation of DNA binding by the yeast glucose transporter gene repressor Rgt I. Mol Cell Biol 2003, 23:5208-52I6.

29. Krivan W, Wasserman WW: A predictive model for regulatory sequences directing liver-specific transcription. Genome Res 200I, I I:I559-I566.

30. Matys V, Fricke E, Geffers R, Gossling E, Haubrock M, Hehl R, Hornischer K, Karas D, Kel AE, Kel-Margoulis OV, et al.: TRANSFAC: transcriptional regulation, from patterns to profiles. Nucleic Acids Res 2003, 3 1:374-378.

3I. Final Motifs [http://fraenkel.mit.edu/Harbison/release v24/ final set/Final InTableS2 v24.motifs]

32. Cavener DR: Comparison of the consensus sequence flanking translational start sites in Drosophila and vertebrates. Nucleic Acids Res 1987, 15:1353-1361.

33. MatrixREDUCE Homepage [http://bussemaker.bio.colum bia.edu/software/MatrixREDUCE/] 
34. The Fraenkel Lab - Harbison et al. Final Motif Logos [http:l /fraenkel.mit.edu/Harbison/release v24/final set/Final motifs/]

35. Su Al, Wiltshire T, Batalov S, Lapp H, Ching KA, Block D, Zhang J, Soden R, Hayakawa M, Kreiman G, et al:: A gene atlas of the mouse and human protein-encoding transcriptomes. Proc Natl Acad Sci USA 2004, 1 0 I:6062-6067.

36. GNF SymAtlas [http://symatlas.gnf.org/SymAtlas/]

37. Wu Z, Irizarry RA: Preprocessing of oligonucleotide array data. Nat Biotechnol 2004, 22:656-658. author reply 658

38. A Model Based Background Adjustment for Oligonucleotide Expression Arrays [http://www.bepress.com/jhubiostat/paperl/]

39. Hubbard T, Andrews D, Caccamo M, Cameron G, Chen Y, Clamp M, Clarke L, Coates G, Cox T, Cunningham F, et al.: Ensembl 2005. Nucleic Acids Res 2005, 33:D447-453.

40. Curwen V, Eyras E, Andrews TD, Clarke L, Mongin E, Searle SM, Clamp M: The Ensembl automatic gene annotation system. Genome Res 2004, 14:942-950.

Publish with Bio Med Central and every scientist can read your work free of charge

"BioMed Central will be the most significant development for disseminating the results of biomedical research in our lifetime. "

Sir Paul Nurse, Cancer Research UK

Your research papers will be:

- available free of charge to the entire biomedical community

- peer reviewed and published immediately upon acceptance

- cited in PubMed and archived on PubMed Central

- yours - you keep the copyright

Submit your manuscript here:

http://www.biomedcentral.com/info/publishing_adv.asp
BiolMedcentral 